\title{
Securing an EHR in a Health Sector Digital Ecosystem
}

\author{
Dr Brian Regan, O. Tolga Pusatli, Eugene Lutton, Dr Rukshan Athauda ${ }^{1}$ \\ ${ }^{1}$ School of Design Communication \& IT, University of Newcastle, NSW, Australia e-mail : brian.regan@newcastle.edu.au
}

\begin{abstract}
This paper examines the challenges in maintaining system security in the dynamic environment of eHealth where there are system components scattered among private and public organisations and used by a spectrum of professionals, each with different needs. The need for more interoperability between these systems has strained the ability of system administrators to maintain compliance with security protocols by staff working in these environments.
\end{abstract}

Index Terms-health, security, privacy, EHR.

\section{INTRODUCTION}

The health sector in all countries is a complex collection of entities that need to interconnect with each other in order to improve patient care by sharing information about individual patients. This is no less the case in Australia where an individual patient can visit a general practitioner (GP), attend a public hospital either as an inpatient or as an outpatient, be taken by ambulance to an emergency room, be treated by a private specialist physician in a private hospital, have $\mathrm{x}$-rays and other imaging done by a private radiologist or be admitted into a private nursing time that receives funding from government as well as the individual. Tracking the history of the patient through all of these elements of the health sector and maintaining a continuous and complete longitudinal information record of their health status has been a challenge for over 30 years. The fact that this challenge is universal is reflected in the UK government establishing the world's largest software project to computerise the NHS [1] and the recent proposal of the US president to invest heavily in improving information systems for the US health system [2]. In the Australian context there has been government projects since the early 1990's to create a comprehensive electronic health record (EHR) however nothing but pilot projects have been implemented.

The fundamental reason why a universal longitudinal EHR has not been developed successfully is largely unrelated to the technology. Once wide-area networks had been introduced the basic technology existed to satisfy the technical requirements of such an EHR. The main obstacles have been professional and organizational barriers within and among the players in the health system that has generated the roadblocks to implementation. The architecture of Web services over the last few years has made the technical obstacles even simpler to address but the organizational barriers still remain. The information systems used in health sectors around the world are examples of complex digital ecosystems with multiple individual players and interacting organisations.

\section{INTEROPERABLE EHR'S}

From the first introduction of IT systems into hospitals in the 1960 's, it was observed that the ability to retain clinical information without loss, distribute it cheaply and rapidly, present it in a clear character set, meant that an electronic version of a patient's health record had many advantages over paper based systems. However, before 1970 the sharing of healthcare data electronically was not seen as necessary because the usage of computers and electronic data was insignificant at that time [3]. In the 1980's distinct departments within hospitals started to use computer systems as tools to manage their local data. However, the systems were still separate from each other and they did not share their data repositories [3]. In the last decade, the system interfaces have given the hope of establishing links among systems for sharing data.

Over time many systems for an electronic health record (EHR) have been developed, implemented and examined. Australia has seen efforts to build a comprehensive EHR system nationwide with common terminology, structure and connectivity since the 1980 's. Many authors have advocated the usefulness of such systems, especially within large tertiary hospitals where patient information has to be shared amongst many health care providers for a single patient and where there were the resources to implement these systems [4-7].

The most significant change to the organisation and funding of health care in Australia came with the introduction of Medibank (later to become Medicare) in 1975 [8]. This was a nationally operated scheme that guaranteed medical care for all citizens by the national government, paying private medical practitioners and providing funds to state governments to fund public hospitals. Medibank followed the existing tradition of paying medical practitioners for each individual service they performed, resulting in the need from the very start to process individual billing transactions for over 100 million medical services delivered each year. Its success on implementation was due to the presence of online transaction processing systems (OLTP) technology.

Since the establishment of a national payment scheme for medical services was objected to by peak medical bodies, the government faced a media campaign that included a scare campaign that the introduction of a "Medibank Num- 
ber" for each person and a central database of medical events would be the basis for government surveillance of citizens. This marked both the first crudely implicit national EHR, but also the start of concern about the privacy of EHR information.

Some of the obstacles to the successful introduction of widespread EHR systems have been identified as the need for appropriate technical skills among health care professionals [9], the lack of a positive business case for their introduction into isolated clinical organisations such as GP clinics [10], also the usability of EHR systems in busy clinical environments [11], and the reluctance of clinical staff to use systems which slow down or obstruct their clinical interaction with the patient [12]. This final obstruction of workflow by the EHR systems is one of the factors we are seeking to address with the RARE protocol described in Section V. Examples of challenges in IT implementation in the health care literature include [13], where the authors note that delays of seconds by clinical systems can provoke resistance to their use by clinical staff.

History has made governments cautious with new projects, because there are many incomplete or collapsed programs so far. "From the Australian experience the establishment of a comprehensive EHR across the health sector requires more than mastering the hardware and software issues, but a greater determinant of success is mastering the workflow issues in the variety of participating organisations." [14]

One of three Australian government pilots of EHR applications to demonstrate possibilities for interoperability was launched as EHR*Net in 2002 [15]. Since renamed as Health eLink, this system allows hospital staff, independent GPs, pathology services and the patient to access summary health information over the Web, with the individual items of the EHR drawn from all the different health organisations that the patient may visit. Currently, Health eLink is being piloted in two NSW sites, Maitland and Greater Western Sydney [16, 17]. A departmental evaluation in September 2008 identified support for this eHealth initiative from clinicians and patients but that the slow adoption of the system was in part due to a lack of "richness of the EHR" provided by Health eLink and that there needs to be increased interoperability with other clinical software [18]. The less acknowledged challenge for these projects and the reason it took 4 years for the initial implementation to be commenced was the concerns over securing the privacy issues of managing this information between the many players involved.

\section{Privacy in Health}

Protecting the privacy of sensitive information held in an EHR relates to the very personal nature of such information, however the variability in attitudes by the public to privacy issues has made protecting such privacy extremely difficult. The essence of this challenge is that individuals want the best of available care and treatment done for them and to achieve this they will agree that any health professional can see their core information in order to assist. At the same time however they will be concerned if their medical condition and history is being discussed outside their team of clinical carers. Defining the barriers and limits to the spread of such information can be extremely difficult. What information does the pathologist need when assessing tissue samples compared to Allied health staff organizing a diet program. Some conventional models have been proposed that have rules such as that psychiatrists need access to all history related to mental illness but the physiotherapist does not. However humans have a complicated relationship with their health information. For example, on receiving news of a possible cancer diagnosis a patient may talk with a complete stranger on the train going home in order to express their frustrations and anxiety, and yet not reveal that same information to close family members for fear of raising their anxiety. Building systems to accommodate such variable behaviour remains a challenge.

The model used for most Australian privacy legislation derives from principles first published by the OECD. Critical principles in this list were the need for consent by an individual for the distribution of their personal information and the importance of appropriate security mechanisms for the databases. The dominance of the consent principle was the major delay in the Health eLink project as lengthy community consultation and government committee discussion was held to get a consensus that patients could be automatically enrolled in the system without consent and only having an opt-out option. The variety of elements in the health ecosystem described in the previous paragraph has driven the national eHealth authority (NEHTA) (established by the national government) to propose "sensitivity labels" on all information such that it becomes important that the specific identity of a user accessing particular data becomes critically important.[19]

\section{ACCess to Health Data}

Providing access to IT resources is a key task for IT administrators to perform (also known as access control configuration) in typical medium to largescale enterprises. With the variety of systems both in the separate departments of hospitals and the private health service providers in the external marketplace, different user groups of professionals and each with different access rights configurations, this task is neither trivial nor straight-forward. Misconfigurations, too excessive or too restrictive access control configurations are common. A direct consequence of excessive access control configurations is access to unauthorised resources. Unauthorised access to information and theft of proprietary information amounts to the largest dollar amount of losses by type [20], as well as compromising privacy control in sensitive areas such as health.

What is an optimal access control configuration? An optimal access control configuration should allow access to only the required resources for authorised users. That is, in optimal access control configurations, (1.) access is not too restrictive to disallow access to required resources for authorised users or (2.) not too excessive allowing access to unauthorised resources.

Too restrictive access control configurations result in usability issues, often disruptive, time-consuming and frustrating users, with additional workload to IT administra- 
tors [21]. Excessively permissive configurations can result in vulnerabilities. A prominent example of excessive permissive configuration is the "Memogate" scandal, where staffers from one political party on the US Senate Judiciary Committee stole the opposing party's confidential memos from a file server that the two parties shared. This was possible in part due to an inexperienced system administrator's error in setting file permissions providing access to unauthorised files [22].

Typically, too restrictive access control configurations are discovered by users when performing tasks and fixed during system use. In contrast, excessive access-control configurations, results in vulnerabilities that may never be detected. Additionally, what is detected could be a small percentage of the actual exploited vulnerabilities! As was in the case of "Memogate", the vulnerability was exploited for over 18 months until detected after the excerpts of the stolen memos began appearing in the press and investigated [22]. It is possible in other cases that such exploited vulnerabilities are never detected. Thus excessively permissive access control systems create vulnerable systems that may be exploited and never detected!

Why has a commonly accepted and used optimal access control configuration not been achieved in practice? To develop an approach to address this question we consider the way that access control rights are configured in practice.

In a typical organisation, there are three main actors that play a role in access control configuration: (1.) the administrator (trusted principal) who configures access control rights; (2.) the employee (principal) who performs authorised tasks; and (3.) typically a manager of the employee who authorises tasks (task authoriser).

Typically information about authorised tasks that need to be performed by employees (principal) is sent to administrators by the respective managers (task authoriser) to the administrator (trusted principal). The IT administrator configures relevant access rights in the different IT systems enabling the user to perform authorised tasks.

The manager (task authoriser) informs the administrator the authorised tasks needed by the employees/users (e.g. generate a cash flow report). The IT administrator is left with the non-trivial role of translating the high-level authorised tasks to low-level access control configurations in the complex mix of various IT systems and networks. To make matters worse, such configuration changes can be frequent in a dynamic environment.

There are a number of reasons for non-optimal access control configurations by administrators as discussed in [23].

* Inherent difficulty in specifying access control requirements: Optimal access control specification requires an understanding of nature of the task, the application systems that these tasks might affect, and the different access right configurations required to perform these tasks in the different configurable systems. It is impractical to expect an administrator to be aware and consider all of these aspects in configuring access rights. Even for a skilled administrator, the possibility for human error is high in such complexity. To exacerbate, often information on tasks may be incom- plete and inconsistent.

* Complexity of today's systems: With a multitude of configurable systems, hundreds of users/roles and a large set of possible permutations, it can be overwhelming for IT administrators to keep track of the different and changing access privilege configurations. For instance, access control rights may need to be granted at various system-levels (e.g. OS level, database-level, application packages, etc.) and it is unrealistic to expect system administrators to be proficient in all these levels and systems.

* Works fine: Unlike other types of configurations, an excessively configured access control system may be undetected both by users and administrators as everything seems to work fine. This makes such non-optimal access configurations harder to detect.

* Unrewarding: Determining optimal access control configurations require both time and effort, but is not demonstrable in functionality or tangible security improvements to management, especially as to our knowledge there are no practical means for verification.

* Unaware of risks of excessively permissive systems: Typically, it is internal users with malicious intent that tend to take advantage of poorly configured excessively permissive access control configurations. The management of such incidents internally has reduced awareness of this as a risk.

* Lack of time and effort for access control configurations: The wide range of system support tasks required of IT administrators means that optimal access control configuration has less focus, lower priority and lacks investment of time and effort.

Methodologies such as discussed in [23] consider means for optimal access control configurations.

\section{USER AUthentication in Health}

While a number of technical solutions exist for achieving user-authentication in IT systems, the common problems with many solutions includes establishing the correct control configuration for each individual user and maintaining user compliance with the protocols imposed to ensure integrity of the authentication.

After three decades of computer systems being deployed into Australian hospital wards and clinics, it is often still accepted practice for many clinical staff to share the same password for the day to avoid going through the time required by login protocols. System administrators may implement any password protocol but the need for efficient turnover in the use of clinical terminals and the mobility of staff around a hospital environment provide motivation for staff to avoid compliance. Such non-compliance can result in problems such as audit trails become of limited value beyond drug ordering as staff only see direct legal consequence for having correct authorisation for prescribing and not for all clinical systems. Thus access to components of the patient records may be open to any member of staff, and any audit trail of access will not be an accurate reflection of what happened. Thus in the context previously mentioned of proposals for labelling and restricting access to "sensitive" clinical information to different staff will become subverted. The "sensitive labels" may address community 
complaints but if compliance is not achieved then it becomes a token measure of appeasement.

The nature of health care and hospital organisation is that delegation to junior staff is usually an option for most IT related actions. The result is avoidance and negative responses by senior clinicians to new IT systems, when it is just these figures who need to set the example for adoption of the systems. The use of shared user accounts means that staff must navigate through the system each time to locate the subset of clinical data relevant to themselves. If staff could sign-on to their own account, then user profile technology could be used to identify and display their patients immediately without the distraction of selecting from a complete ward list. With clinical staff in emergency rooms involved in over 30 events per hour and being interrupted more than 11 times per hour, the need to be able to abandon and then return to the mid-point of a computer interaction would avoid common inefficiencies [24].

\section{IMPROVING AUTHENTICATION COMPLIANCE}

Thus an authentication mechanism that makes minimal impact on the workflow of clinical staff will not only encourage the formation of a valid audit trail within clinical systems but also enhance the usability of the systems and encourage their active adoption by staff.

The RFID Authentication for Repeat Entry (RARE) security protocol, requires staff to login to the organisation's network at the beginning of their working day on any authorised computer on the network. For the remainder of that person's working shift, they would wear a normal staff identity badge (as is common practice in all Australian health care facilities) that in addition to a photo and name details would contain a passive radio frequency ID tag (RFID). Whenever they approach, within a predetermined range of a computer on the organisation's network, then their particular profile as a user would be activated on the computer adjacent to them. After a predetermined length of time considered adequate to cover the normal working time for that user, then they would be automatically logged off the network. A variation on this protocol was also presented in [25].

At any stage during a shift where an RFID badge is lost or stolen, a call to the system help desk could log that account off for the shift and invalidate the badge ID from further use. Badges lost or stolen from employees in their homes or other location after work could not be used without a matching password, the only vulnerability would require the physical theft of an employees badge during a shift in the hospital grounds, which for the perpetrator represents a high risk action merely for access to clinical records.

This represents a compromise in terms of security. It is trading off a fully robust security solution with gaining a usable compliance by clinical staff. It also represents a trade-off of security against improved productivity in terms of the staff time expended in clicking through sign-on windows and tracking through menu choices to reach a point they were at when they last were using the computer.

\section{RFID TECHNOLOGY}

The RARE protocol is based on Radio Frequency Identification System (RFID) that is worn by a computer user. "RFID tags are similar to smart cards in that they carry information or data that the tag or transponder exchange information using electromagnetic fields" [26] p6). RFID has its origins in radio broadcast technology and radar, and these two disciplines commenced research in identification of objects using radio frequencies [27]. Radar detection was used by the defence forces during World War 2 and later developed for use in RFID systems [27, 28]. The first paper written on the subject of RFID was by Harry Stockman, "Communication by Means of Reflected Power", which gave the idea of RFID but was not feasible due to limitations of the available technology at that time [27-29]. Landt states that it would not be till the 1970's before it was possible to implement the idea that Stockman had written about in 1948. During the 1970's applications were developed for animal tracking and electronic toll collection though the later was not ready for some years for commercial implementation [27]. However in each of these environments, the implementation has to confront issues of the physical environment [23]. Animal tags need to cope with poor reading rates from mud and water, while toll collection systems can be confused multiple reads by adjacent readers.

\section{IMPLEMENTATION ISSUES}

The key to implementing RARE in most operating environments is to use Fast User Switching (FUS), which has been incorporated into a number of operating systems including the Windows XP platform that was used for developing a RARE prototype. FUS allows multiple connections (user sessions) to be run on the same computer at the same time. It maintains the state of a user's session, while another user can also logon. Thus the first user does not need to close their applications and log off the computer. Each user has a profile and a secure desktop. To use FUS a user has to logon to a computer. Multiple users can be logged on to a computer and their account (programs) kept active even though they do not have control of the current desktop [30].

When the computer starts the welcome screen is shown and a user clicks on their account icon and then is prompted to enter their user identification and password. This is completed on the Winlogon desktop, which only allows Winlogon to be active. Winlogon authenticates the user and Gina sends the token and profile information to Winlogon. If the user is currently logged on then Winlogon switches the user to their previous desktop. Winlogon will create a new thread if the user is not logged on and the thread creates a new desktop and starts the user shell on the desktop. The thread and desktop information is added to the list of users connected to the computer. Now the Winlogon desktop is switched to the user desktop and the user can now use the computer.

If the user wishes to $\log$ off, the user thread will detect the log off event and the user thread will finish. The applications of the user are still active but since the desktop is "switched out" the user has no ability to interact with them. 
The welcome screen will displays the accounts of the users and can show if any emails have arrived or how many programs are running on the switched out user account. Now if the user wishes to reactivate their "switched out" account they $\log$ back in and are now able to return to the programs they were using previously. It is at this point that the RFID reader attached to the computer can be used to initiate a message to Gina to logon to the session again or alternatively if the RFID reader's polling detects the withdrawal of the RFID tag from reading range then again the user's thread is switched out.

One problem with Fast User Switching (FUS) is that if a user shuts down the computer all work that is not saved by any logged on user is lost. This means all other logged on users are also affected by the action of one user. Another issue is that FUS is not available if the computer is part of a domain. This lack of domain functionality will hinder the implementation within a networked hospital system. One reason why this occurs is that the users on the computers have to be included in list of users connected to the computer, which would be a problem if that list contained hundreds of accounts. The architecture was designed for a small number of accounts on the computer. Though for this research we are looking at using this ability in a hospital environment where more than one staff member uses the same computer in the ward, it will be possible to implement a pilot system in a single ward if the number of users is limited to only clinical staff assigned to that ward.

FUS has provided a prototyping environment but for an implementation covering a large networked organisation such as a hospital then a different operating system tool will be required.

\section{Conclusion}

This paper has proposed a security protocol that is suitable for the dynamic digital ecosystem of major health establishment such as teaching hospital. The need to properly secure information in health sector organisations has up till now been subsumed beneath the first requirement of building effective and utilised EHR systems. As these systems are now becoming a reality to convey critical information across the multiple components of the health system, it is important to have a security protocol that will not only be effective but also gain user compliance.

\section{REFERENCES}

[1] UK National Audit Office, "The National Programme for IT in the NHS: Progress since 2006," UK National Audit Office, London 16 May 20082008.

[2] "American Recovery and Reinvestment Act of 2009," 111th Congress ed USA, 2009, p. 163.

[3] J. T. Demetriades, "Informatics Development - Technologies for Integration," in HIC, Melbourne, 1996.

[4] J. H. v. Bemmel, Handbook of Medical Informatics: Springer-Verlag, 1997.

[5] B. I. Blum, Information systems for patient care. New York: Springer-Verlag, 1984.

[6] R. S. Dick and E. B. Steen, The computer-based patient record: an essential technology for health care. Washington, DC: National Academy Press, 1991.
[7] S. Levey and L. N. Paul, Health care administration |ba managerial perspective. Philadelphia, 1973.

[8] R. B. Scotton and C. R. Macdonald, The Making of Medibank Kensington, N.S.W. : School of Health Services Management, UNSW, 1993.

[9] M. Tsiknakis and A. Kouroubali, "Organizational factors affecting successful adoption of innovative eHealth services: A case study employing the FITT framework," International Journal of Medical Informatics, vol. 78, pp. 39-52, January.2009 2009.

[10] J. Knight, M. Patrickson, and B. Gurd, "Towards Understanding Apparent South Australian GP Resistance to Adopting Health Informatics Systems," in 19th Australasian Conference on Information Systems (ACIS), Christchurch, New Zealand, 2008, pp. 492-501.

[11] P. J. Edwards, K. P. Moloney, J. A. Jacko, and F. Sainfort, "Evaluating usability of a commercial electronic health record: A case study," International Journal of Human-Computer Studies, vol. 66, pp. 718728, October.2008 2008.

[12] J. S. Ash and D. W. Bates, "Factors and forces affecting EHR system adoption: Report of a 2004 ACMI discussion," Journal of the American Medical Informatics Association, vol. 12, pp. 8-12, JanuaryFebruary.2005 2005

[13] A. Georgiou, A. Ampt, N. Creswick, J. I. Westbrook, and J. Braithwaite, "Computerized Provider Order Entry-What are health professionals concerned about? A qualitative study in an Australian hospital," International Journal of Medical Informatics, vol. 78, pp. 60-70, January.2009 2009.

[14] B. Regan and O. T. Pusatli, "Challenges in Producing a National Comprehensive Electronic Health Record in Australia," in International Symposium on Health Informatics and Bioinformatics (HIBIT), Antalya, Turkey, 2005, pp. 150-156.

[15] EHR Working Group:A Working Group of the Information Management Implementation Coordination Group (IMICG), NSW Health Strategy for the Electronic Health Record NSW EHR*Net: NSW Health Department, 2002.

[16] Electronic Health Record, "EHR * Net Projects." vol. 2005, 2003.

[17] Electronic Health Record, "EHR*Net Strategy - health e link." vol. 2005, 2004.

[18] KPMG, "An Evaluation of the Healthelink Electronic Health Record Pilot," Summary Report September.2008 2008.

[19] NEHTA, "Privacy Blueprint for the Individual Eelectronic Health Record - Report on Feedback," National E-Health Transitition Authority, Sydney 7/11/2008 2008 .

[20] M. P. L. L. A. Gordon, W. Lucyshyn, and R. Richardson, CSI/FBI Computer Crime and Security Survey: Computer Security Institute, 2005.

[21] L. Bauer, S. Garriss, and M. K. Reiter, "Detecting and Resolving Policy Misconfigurations in Access-Control Systems," in Proceedings of the ACM Symposium on Access Control Models and Technologies, Estes Park, CO, USA, 2008, pp. 185-194.

[22] U. S. Senate Sergeant at Arms, "Report on the investigation into improper access to the Senate Judiciary Committee's Computer System," US Senate, Washington March 4th, 20042004.

[23] R. Athauda, G. Skinner, and B. Regan, "A Methodology to Minimise Excessively Permissive Security Configurations," in Proceedings of the 8th WSEAS International Conference on Applied Computer Science $\left(A C S^{\prime} 08\right)$, Venice, Italy, 2008, pp. 187-192.

[24] E. W. Coiera, R. A. Jayasuriya, J. Hardy, A. Bannan, and M. E. Thorpe, "Communication loads on clinical staff in the emergency department.[see comment]," Medical Journal of Australia, vol. 176, pp. 415-8, May 6, 20022002.

[25] Q. Wang and H. Jin, "Usable Authentication for Electronic Healthcare Systems," in SOUP 2008 Pittsburgh,PA, 2008.

[26] K. Finkenzeller, RFID Handbook: Fundamentals and applications in contactless smart cards and identification. New York: John Wiley and Sons, Inc, 2003.

[27] J. Landt, "Shrouds of Time, The history of RFID," AIM Inc. 2001.

[28] A. Weiss, "RFID tags polarize the debate over privacy vs. efficiency," netWorker, vol. 7, pp. 24-30, September 2003.

[29] Stockman, "Communication by Means of Reflected Power," in IRE, 1948, pp. 1196-1204.

[30] Microsoft, "Architecture of Fast User Switching." vol. 24/10/2004, 2001. 